\title{
Measuring Closest Water, Sanitation, and Hygiene Facilities in Unhoused Communities of Los Angeles, USA
}

\author{
Lourdes Johanna Avelar Portillo ${ }^{\mathrm{a}, *}$, Lois Park ${ }^{\mathrm{a}}$, Charlene Ko ${ }^{\mathrm{a}}$, Angelica Vasquez ${ }^{\text {a }}$, Meredith \\ Franklin $^{\mathrm{a}}$, Yao-Yi Chiang ${ }^{\mathrm{b}}$ \\ ${ }^{a}$ University of Southern California, avelarpo@usc.edu,Lois Park, loispark@usc.edu, Charlene Ko, charlejk@usc.edu,Angelica \\ Vasquez,vasq313@usc.edu, Meredith Franklin,meredith.franklin@usc.edu \\ ${ }^{b}$ University of Minnesota,yaoyi@umn.edu \\ * Corresponding author
}

Keywords: Closest Facility, Network Analysis, GIS, Water Insecurity, WaSH, SDGs, Homelessness, Los Angeles, Urban Populations, Unhoused Communities

\begin{abstract}
:
According to the World Health Organization and the United Nations, more than 2 billion people worldwide lack safe access to water for drinking, sanitation, and hygiene (WaSH) services. The inaccessibility of these essential services contributes to $\mathrm{WaSH}$ insecurity, which creates health disparities and disproportionately increases the risk of preventable infectious diseases. WaSH insecurity significantly affects communities in the global South. However, vulnerable communities in the United States (US) receive limited attention due to the misconception that universal access to WaSH exists in wealthy nations. Thus, the goal of this study is to understand how WaSH insecurity impacts communities experiencing homelessness in Los Angeles (LA), California through the lens of Sustainable Development Goals 6 and 11 that aim to ensure access to water and sanitation, and affordable housing for all, respectively.
\end{abstract}

According to the Los Angeles Homeless Services Authority (LAHSA), on a single night in January 2020, it is estimated that 66,436 people are experiencing homelessness, $72 \%$ of whom live in unsheltered conditions. Worse yet, these estimates have likely risen due to the impacts of the SARS-CoV2 (COVID-19) pandemic. The accessibility of essential WaSH services determines health outcomes, but people experiencing homelessness live at the margins of society with limited access to drinking water, restrooms, showers, and laundry services needed to sustain people's livelihoods. To this end, for people living in unsheltered environments, the global pandemic has made a dire situation worse with the closure and reduction of publicly available services.

This study explores survey data collected from unhoused individuals in Los Angeles to understand the impacts of urban WaSH insecurity. By combining surveys with road network data, this study integrates geographic information systems (GIS) to measure the distance of WaSH facilities available in the community to people's reported sleeping locations using closest facility analysis. The results from the GIS models were then imported into R and compared to the facilities people reported using on a daily basis. The findings of this study reveal the inconsistency between the closest facility distance measured in the GIS models and actual places people chose to use. In other words, people do not always choose to use the facility that is closest to them. In some cases, people travel as far as 16 miles (or $25 \mathrm{~km}$ ) to access shower or laundry facilities. At the same time, this study highlights that the importance of distance as a decision factor varies depending on the time of day and service type.

The inaccessibility to secure housing and WaSH services forces people to engage in survival strategies that increases the risks of adverse health outcomes in an already vulnerable community. This study demonstrates that non-profit organizations contribute the most to filling gaps in WaSH services. Data from the study suggest that more practical policies can effectively address the needs of unhoused communities, including providing WaSH services that are safe, affordable, and available 24-hours. These findings are significant in that they provide the foundation for research-driven policies aimed at reducing the risk of infectious diseases, improving people's health, and increasing the safety of urban communities. 